\title{
FL: Genmutationen im Prognosescore ergänzen
}

Obwohl follikuläre Lymphome genetisch heterogen sind, wurde die prognostische Aussagekraft somatischer Mutationen noch nicht systematisch erfasst. Dies holten Forscher nun nach.

$B^{e}$ 151 Patienten mit follikulärem Lymphom (FL) aus der Phase-III-Studie GLSG 2000 wurde in Biopsien (max. 1 Jahr vor Beginn der R-CHOP-Erstlinientherapie entnommen) retrospektiv der Mutationsstatus von 74 Genen analysiert. In multivariaten Risikomodellen erschien der neue Score m7-FLIPI geeignet,

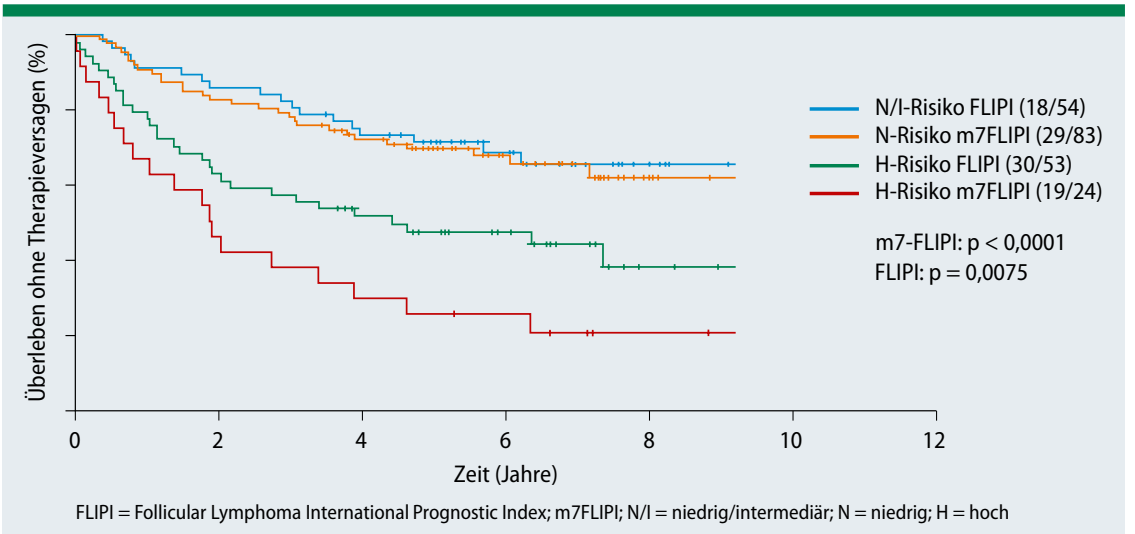

Abb. 1: Der m7-FLIPI hat eine bessere prognostische Aussagekraft als der FLIPI.

\section{Beim Mantelzelllymphom Ansprechen nach Stammzelltransplantation erhalten}

\section{Eine Hochdosistherapie gefolgt von einer autologen Stammzelltransplanta- tion (ASCT) führt bei den meisten Patienten mit einem Mantelzelllymphom (MCL) zu einem objektiven Ansprechen, aber die Rezidivraten sind hoch.}

aut Zwischenergebnissen einer PhaseL III-Studie kann eine Erhaltungstherapie mit Rituximab (MR) das progressionsfreie Überleben (PFS) von MCL-Patienten verbessern. Bis die endgültigen Resultate vorliegen, ist der Effekt auf das Gesamtüberleben (OS) aber noch unklar, weshalb Forscher retrospektiv $157 \mathrm{MCL}$ Patienten analysierten, die sich an ihrem Zentrum einer ASCT unterzogen hatten. 50 hatten eine MR erhalten (32\%), 107 nicht (68\%). Alle Patienten der MRGruppe hatten zuvor schon Rituximab bekommen, in der Vergleichsgruppe dagegen 13 Patienten noch nicht $(p=0,01)$. Deutlich häufiger waren die Patienten der MR-Gruppe zuvor u.a. auch mit Hochdosis-Cytarabin als Teil der Induktionstherapie behandelt worden.

Eine MR war mit einem besseren PFS gegenüber keiner MR assoziiert (Hazard Ratio [HR] 0,44, $95 \%$-Konfidenzintervall [95\%-KI] 0,24-0,80; $\mathrm{p}=0,007)$. Das resultierte in der multivariaten Auswertung nach median etwa 5 Jahren auch in einem besseren OS (HR 0,46, 95\%-KI ptomatischem FL validiert, die eine Erstlinientherapie aus R-CVP erhielten.

In der Trainingskohorte differenzierte der m7-FLIPI eine Hochrisiko(HR)Gruppe (28\%) von einer Niedrigrisiko (NR)-Gruppe (5-Jahres-,,failure free survival“ [FFS] 38,29 vs. 77,21 \%; Hazard Ratio [HR] 4,14; $\mathrm{p}<0,0001)$. Der m7-FLIPI übertraf ein Prognosemodell, das nur die Genmutationen berücksichtigte (HR $3,76)$. Auch in der Validierungskohorte definierte der m7-FLIPI eine HR- (22\%) und NR-Gruppe (5-Jahres-FFS 25 vs. $68,24 \%$; HR 3,58; $\mathrm{p}<0,0001$ ). In der Validierungskohorte verbesserte sich die $\mathrm{Ri}$ sikostratifizierung durch den m7-FLIPI versus dem alleinigen FLIPI (HR 2,18) (Abb. 1) und der Kombination aus FLIPI und ECOG-Performancestatus (HR 2,03).

Fazit: Die Integration des Mutationsstatus von 7 Genen in einen klinisch geprägten Risikoscore verbesserte die prognostische Aussagekraft bei FL-Patienten unter einer konventionellen Erstlinien-Immunchemotherapie. Brigitte Schalhorn

Pastore A et al. Integration of gene mutations in risk prognostication for patients receiving firstline immunochemotherapy for follicular lymphoma: a retrospective analysis of a prospective clinical trial and validation in a populationbased registry. Lancet Oncol. 2015;16(9):1111-22.

0,23-0,93; $\mathrm{p}=0,03$ ). Der OS-Gewinn wurde wesentlich durch die Zeit bis zur Progression hervorgerufen, das Überleben nach Rezidiv war in den Gruppen vergleichbar lang. In der MR-Gruppe traten mehr Neutropenien vom Grad 3/4 auf ( 34 vs. $18 \%, p=0,04$ ), aber die MR beeinflusste die nicht mit einem Rezidiv assoziierte Mortalitätsrate nicht.

Fazit: Es zeigte sich bei den MCL-Patienten durch die MR eine Verbesserung des PFS. Dies scheint auch in einen Überlebenszeitgewinn zu münden. Der generelle Einsatz einer solchen Erhaltungstherapie sollte aber erst nach Bestätigung der Ergebnisse in prospektiven randomisierten Studien erfolgen.

Friederike Klein

Graf SA et al. Maintenance rituximab after autologous stem cell transplantation in patients with mantle cell lymphoma. Ann Oncol. 2015;26(11): 2323-8. 